\title{
Avaliação da frequência cardíaca materna, fetal e neonatal e sua variabilidade em equinos da raça Paint Horse ${ }^{1}$
}

\author{
Angélica Alfonso², Raíssa K.S. Cruz², Dario A. Cedeño Quevedo ${ }^{3}$, \\ Carlos Roberto Padovani ${ }^{4}$, Roberto C. Gonçalves ${ }^{2}$, Simone B. Chiacchio ${ }^{2}$ \\ e Maria Lúcia G. Lourenço ${ }^{2 *}$
}

\begin{abstract}
Alfonso A., Cruz R.K.S., Cedeño Quevedo D.A., Padovani C.R., Gonçalves R.C., Chiacchio S.B. \& Lourenço M.L.G. 2018. [Maternal, fetal and neonatal heart rate evaluation and its variability in the Paint Horse breed.] Avaliação da frequência cardíaca materna, fetal e neonatal e sua variabilidade em equinos da raça Paint Horse. Pesquisa Veterinária Brasileira 38(3):551-557. Departamento de Clínica Veterinária da Faculdade de Medicina Veterinária e Zootecnia, Universidade Estadual Paulista, Botucatu, SP 18618-687, Brazil. E-mail: mege@fmvz.unesp.br

The aim of this study were to describe the heart rate (HR) and indexes of maternal and fetal heart rate variability (HRV) in final period of pregnancy, as well as to describe the evolution of the development of autonomic nervous system during fetal and neonatal period. There were 20 animals in each category, whose maternal and fetal electrocardiographic examinations were performed at 15 and 7 days antepartum. Neonatal ECG it was evaluated at birth until the first 48 hours of life, and then once a week up to 35 days. There were significant differences in fetal heart rate (FHR) during this period, but the fetal HRV indexes have not changed. There were no significant differences in the rates of maternal HRV. The mean of fetal HR decreased significantly from 15 to seven antepartum days $(95.6 \pm 11.4 \mathrm{bpm} ; 83.1 \pm 12.6$, respectively), though the fetal HRV indexes have not decreased. The results of fetal and neonatal HRV in the present study, when compared to maternal indicate the parasympathetic dominance during fetal and neonatal sympathetic phase during to the third and/or fourth weeks of age, at which point begins the modulation of the two systems.

INDEX TERMS: Heart rate, horses, Paint Horse, electrocardiogram, neonatal hypoxia, foals, autonomic nervous system, physiology.
\end{abstract}

RESUMO.- Os objetivos deste estudo foram descrever a frequência cardíaca (FC) e os índices de variabilidade da frequência cardíaca (VFC) materna e fetal no terço final da gestação, bem como descrever a evolução do desenvolvimento do sistema nervoso autônomo durante o período fetal e neonatal. Foram avaliados 20 animais de cada categoria, cujos exames eletrocardiográficos, maternos e fetais, foram realizados aos 15 e sete dias pré-parto. Quanto ao eletrocardiograma

\footnotetext{
${ }^{1}$ Recebido em 25 de setembro de 2015.

Aceito para publicação em 25 de abril de 2017.

Pesquisa de Mestrado com apoio CAPES.

2 Departamento de Clínica Veterinária, Faculdade de Medicina Veterinária e Zootecnia, Universidade Estadual Paulista (Unesp), Botucatu, SP 18618-687, Brasil. *Autor para correspondência: mege@fmvz.unesp.br

${ }^{3}$ Departamento de Saúde Animal, Faculdade de Ciências Pecuárias, Universidade de Nariño, Pasto, Colombia.

${ }^{4}$ Departamento de Bioestatística, Instituto de Biociências, Universidade Estadual Paulista, Botucatu, SP 18618-687, Brasil.
}

neonatal, os momentos avaliados foram ao nascimento até as primeiras 48 horas de vida, e posteriormente, uma vez por semana até os 35 dias de idade. Ocorreram diferenças significativas na frequência cardíaca fetal (FCF) no período avaliado, porém os índices de VFC fetais não se alteraram. Não foram encontradas diferenças significativas nos índices de VFC materna. A média da FCF diminuiu significativamente dos 15 para sete dias do pré-parto $(95,6 \pm 11,4 \mathrm{bpm} ; 83,1 \pm 12,6$, respectivamente), entretanto os índices de VFC fetal não diminuíram. Os resultados obtidos da VFC fetal e neonatal deste estudo, quando comparados aos maternos, indicaram predomínio parassimpático durante a fase fetal e, simpático durante a neonatal, até a terceira e/ou quarta semanas de idade, momento no qual se inicia o equilíbrio entre os dois sistemas.

TERMOS DE INDEXAÇÃO: Frequência cardíaca, equinos, Paint Horse, eletrocardiograma, hipóxia neonatal, potros, sistema nervoso autônomo, fisiologia. 


\section{INTRODUÇÃO}

A compreensão das consequências cardiovasculares da prenhez é fundamental para o manejo de fêmeas prenhes e de seus fetos (Dennis et al. 2010). As observações clínicas confirmam que ocorreram mudanças, embora a natureza precisa destas alterações não sejam bem evidenciadas na literatura, em equinos. A hipóxia é o principal distúrbio relacionado à prenhez, podendo comprometer a viabilidade fetal. A oxigenação do feto depende do aporte sanguíneo adequado para a placenta, e a diminuição da atividade cardíaca fetal é a única possibilidade de se reduzir o consumo de oxigênio. A resposta primária à hipóxia fetal é, portanto, a diminuição dos batimentos cardíacos. Os movimentos fetais estão associados a aumentos transitórios da frequência cardíaca, logo a ausência destes episódios sugerem baixa atividade fetal e comprometimento do mesmo (Adams-Brendemuehl \& Pipers 1987, Manning 2002, Bocking 2003).

De acordo com a teoria da hipóxia gradual (Vintzileos et al. 1991), o primeiro parâmetro a se mostrar alterado diante de baixas concentrações de oxigênio é o controle autonômico (taquicardia e bradicardia) e, apenas posteriormente, há diminuição dos movimentos respiratórios, corporais e tônus muscular. Esta teoria defende que quanto mais tardio for o amadurecimento de determinado órgão, mais sensível é à hipóxia.

A frequência cardíaca (FC) é um importante parâmetro de bem-estar fetal, sendo a sua monitoração útil na detecção de alterações relacionadas à viabilidade fetal (Adams-Brendemuehl \& Pipers 1987). No entanto, a função cardiovascular pode ser avaliada na égua prenhe e no feto não apenas por este parâmetro, mas também, pela análise da variabilidade da frequência cardíaca (VFC), isto é, flutuações em curto prazo na frequência cardíaca. A VFC reflete a influência antagonônica oscilatória dos ramos simpático e parassimpático (vagal) do sistema nervoso autônomo (SNA) sobre o nodo sinusal. Avaliação da VFC permite a análise da resposta do SNA frente ao estresse, e reduções nos seus valores indicam dominância simpática, enquanto o aumento se correlaciona com elevado tônus parassimpático (Von Borell et al. 2007). Nos seres humanos, a VFC fetal aumenta acentuadamente após 30 semanas de gestação, refletindo o desenvolvimento e atuação do SNA sobre o controle da atividade cardíaca (Wheeler et al. 1979, Van Leeuwen et al. 1999). Valores de VFC reduzidos são considerados sinais de comprometimento fetal (Dawes et al. 1992).

Em neonatos, o sistema autonômico não está completamente maduro, ocorrendo poucas variações nos ciclos sinusais frente às alterações diárias, como estresse e repouso. Esse fato é demonstrado com a alta FC logo após o parto e que perdura durante semanas até atingir valores semelhantes aos adultos. A VFC sofre influência da circulação sistêmica e periférica, débito cardíaco e desenvolvimento do nodo sinusal, tornando o período neonatal muito importante para a maturação do sistema cardiovascular que suprirá as necessidades hemodinâmicas adequadamente quando adultos (Woods et al. 1977, Siimes et al. 1990). Os valores normais de VFC em potros, no entanto, são desconhecidos e não se sabe em que momento do período neonatal ocorre a maturação e equilíbrio entre os ramos do sistema nervoso autônomo na espécie equina.

A VFC pode ser calculada usando o traçado eletrocardiográfico com base na medida dos intervalos RR individualmente e na comparação entre RR adjacentes (Brito 2009, Pereira 2011), sua análise é composta por índices obtidos por meio de métodos lineares, como no domínio do tempo e da frequência, e métodos não lineares (Aubert et al. 2003). No estudo do domínio do tempo, assim denominado por expressar os resultados em milissegundos, mede-se cada intervalo RR normal (batimentos sinusais) durante determinado intervalo de tempo e, a partir daí, com base em métodos estatísticos ou geométricos (média, desvio padrão e índices derivados do histograma ou do mapa de coordenadas cartesianas dos intervalos RR), calculam-se os índices tradutores de flutuações na duração dos ciclos cardíacos (Task Force 1996, Pumprla et al. 2002)

Em equinos, a FC e a variabilidade da frequência cardíaca (VFC) do feto podem ser obtidas pelo eletrocardiograma (ECG) materno-fetal transcutâneo, revelando-se um método de fácil realização a campo em grandes animais (Nagel et al. 2010, 2011, 2012, 2014, Baska-Vincze et al. 2015, Trenk et al. 2015).

0 acompanhamento da frequência cardíaca fetal permite não apenas a verificação da vitalidade e viabilidade do feto, mas também fornece informações importantes sobre o estágio de desenvolvimento do SNA. A obtenção dos valores de VFC fetal em condições a campo, bem como a descrição dos índices em animais saudáveis oriundos de partos eutócicos é importante, uma vez que estudos sobre o assunto na espécie são escassos. Assim, o objetivo deste estudo foi descrever os valores de FC e índices de VFC materna e fetal a fim de se determinar se há alterações nesses parâmetros, capazes de indicar a proximidade do parto, e descrever a maturação do SNA fetal e neonatal em equinos da raça Paint Horse.

\section{MATERIAL E MÉTODOS}

O estudo foi realizado no Haras Escaramuça, no Município de Avaré, Estado de São Paulo, 766 metros de altitude, latitude de $23^{\circ} 05^{\prime} 55^{\prime \prime}$, longitude de $48^{\circ} 55^{\prime} 3^{\prime \prime}$, apresentando clima subtropical. Todos os procedimentos experimentais deste estudo foram realizados após aprovação na Comissão de Ética no Uso de Animais da Faculdade de Medicina Veterinária e Zootecnia da Universidade Estadual Paulista "Júlio de Mesquita Filho", campus Botucatu, sob protocolo no 231/2012-CEUA. 0 termo de consentimento livre esclarecido foi assinado pelo proprietário.

Foram avaliados 20 potros da raça Paint Horse, nascidos em eutocia, em partos não gemelares. A data da prenhez foi calculada a partir da data da ovulação, contados 11 meses a partir desta. As éguas receptoras foram vacinadas com Pneumabort- $\mathrm{K}^{\circledR}+1 \mathrm{~b}$ (Rinopneumonite equina, Zoetis Animal Health Specials, Canadá) no quinto, sétimo e nono mês de prenhez. Os animais foram mantidos em pastagem de Tifton 85 (Cynodon dactylon), com sal mineral à vontade e ração comercial com $17 \%$ de proteína $(2,0 \mathrm{~kg} /$ animal/dia). Todas as éguas passaram por exame clínico geral (temperatura, coloração de mucosas, frequência cardíaca e respiratória), e encontravam-se 
saudáveis durante presente o estudo. Os partos foram assistidos e ocorreram sem complicações, não sendo necessária nenhuma intervenção obstétrica.

Os exames eletrocardiográficos materno-fetal foram realizados com as éguas mantidas em bretes de contenção, porém sem causar estresse aparente ao animal. A duração da gravação dos exames foi de cinco minutos, sempre se iniciando pelas manhãs (por volta das 8 horas), sendo realizadas em dois momentos da prenhez, aos 330 e aos 338 dias. Para a realização do eletrocardiograma materno e neonatal foi empregado aparelho computadorizado (ECG PC-TEB, Tecnologia Eletrônica Brasileira, São Paulo) nas seis derivações de membros (I, II, III, aVR, aVL e aVF), na velocidade de $50 \mathrm{~mm} / \mathrm{seg}$ e sensibilidade ajustada para $1 \mathrm{~cm}=1 \mathrm{mV}$, ao longo de um minuto.

Para o exame eletrocardiográfico fetal, o aparelho utilizado correspondeu ao mesmo dos exames maternos. Entretanto, para a fixação dos eletrodos, foram utilizados eletrodos adesivos a base de hidrogel, de acordo com o seguinte posicionamento: eletrodo verde na tábua do pescoço do lado esquerdo; eletrodo amarelo na região do flanco esquerdo, eletrodo preto na região da garupa e, eletrodo vermelho na região do abdômen direito posicionado acima e caudal à região do colón ventral direito (Nagel et al. 2010). Para cada registro eletrocardiográfico, foi analisada a frequência cardíaca fetal, durante cinco minutos.

Para cada registro eletrocardiográfico materno, fetal e neonatal, avaliou-se a frequência cardíaca média, duração do intervalo RR e a VFC no domínio do tempo, por meio da análise de intervalos RR consecutivos (em milissegundos), a partir dos quais se calculou a variância amostral, cujo logaritmo natural (neperiano) representava o índice de tônus vasovagal (iTVV) (Häggström et al. 1996, Tárraga et al. 2000, Carareto et al. 2007), o RMSSD (raiz quadrada da média do quadrado das diferenças entre intervalos RR normais adjacentes) e o RMSM (raiz quadrada da somatória do quadrado das diferenças dos valores individuais em relação ao valor médio, dividido pelo número de iR-R em 90 segundos - VFC global) (Siimes et al. 1990). A escolha desses índices foi feita por tratar-se de indicadores sensíveis e convencionalmente aceitos para caracterização da VFC (Task Force 1996). (Fig.1)

$i T V V=\log _{e}\left(\frac{\sum_{i=1}^{N} R R_{\mathrm{i}}^{2}-\left(\sum_{i=1}^{N} R R_{\mathrm{i}}\right)^{2}}{N(N-1)} R M S S D=\sqrt{\frac{\sum_{i=1}^{N-1}\left(R R_{i}-R R_{i+1}\right)^{2}}{N-1}} \quad R M S M=\sqrt{\frac{\sum_{t=1}^{N}\left(R R_{i}-\overline{R R}\right)^{2}}{N}}\right.$

Fig.1. Fórmulas utilizadas para o cálculo da $\operatorname{VFC}\left(\sum=\right.$ somatória, $\mathrm{RR}$ = intervalos $\mathrm{RR}, \mathrm{N}$ = números de intervalos $\mathrm{RR}$ na série selecionada, loge = logaritmo natural neperiano).
Imediatamente após o parto (ao nascimento e 10 minutos após), todos os potros foram examinados, segundo escore de Apgar (Smith 2006) (Quadro 1) e verificou-se que os mesmos estavam maduros e saudáveis e capazes de permanecerem em pé e de mamar, dentro do intervalo de tempo estabelecido para a espécie e de acordo com os padrões de normalidade (Nogueira \& Lins 2009). As gravações do ECG no período neonatal ocorreram: ao nascimento, quatro, oito, 12, 16, 20, 24, 36 e 48 horas pós-parto, e posteriormente uma vez por semana até os 35 dias de idade, perfazendo um total de 16 momentos. Durante as gravações os potros permaneceram junto de suas mães em piquetes de parto individuais, ou baias individuais.

0 registro foi feito com os animais contidos em local dotado de piso revestido com placas de borracha, de modo a evitar interferências no traçado eletrocardiográfico. Os animais foram mantidos em estação, sem qualquer tipo de sedação, tranquilização ou anestesia, e o eletrocardiograma foi realizado durante no mínimo cinco minutos.

$\mathrm{Na}$ análise estatística, as variáveis dependentes (FC, intervalo RR e índices da VFC) com medidas repetidas no tempo foram submetidas ao teste de normalidade de Shapiro-Wilk. Posteriormente, foi realizado a ANOVA através do PROC MIXED do SAS (SAS, Inst. Inc., Cary, NC, EUA). Fontes de variação no modelo incluindo tratamento nos momentos de avaliação materna e fetal (aproximadamente 15 dias e sete dias pré-parto) e neonatal (ao nascimento, quatro, oito, $12,16,20,24,36,48$ horas após o parto, sete, 14, 21, 28 e 35 dias de idade), e interações de primeira ordem foram consideradas como efeitos fixos. Para todas as análises o nível de significância adotado foi de $5 \%$.

\section{RESULTADOS}

Os índices de VFC materna não se alteraram previamente ao parto (15 e sete dias), embora apresentassem aumento discreto da FC e do RMSSD e redução nos valores do iTVV, intervalo RR e RMSM. Houve diferença significativa dos parâmetros maternos quando comparados aos fetais e neonatais, sendo os maternos, superiores em todos os momentos da análise.

Nos valores fetais para equinos da raça Paint Horse, notou-se decréscimo significativo da FC entre 15 e sete dias $(95,6 \pm 11,4 \mathrm{bpm} ; 83,1 \pm 12,6 \mathrm{bpm}$, respectivamente) ( $\mathrm{p}=0,0413)$ (Fig.2). Os demais índices da VFC fetal (intervalo RR, RMSM, RMSSD, iTVV) (Fig.3-5), não se alteraram previamente ao parto. Os parâmetros fetais diferiram estatisticamente dos maternos, nos dois momentos de análise, e também dos parâmetros neonatais desta raça a partir do nascimento, exceto nas últimas semanas do período neonatal (21, 28 e 35 dias), quando alguns índices, nos neonatos, foram semelhantes aos fetais. A FC e o iTVV fetal aos 15 dias, não diferiram significativamente dos encontrados em neonatos a partir do $28^{\circ}(\mathrm{p}=0,4853 ; \mathrm{p}=0,3421)$ e no $35^{\circ}$ dia de idade ( $\left.p=0,6009 ; p=0,2721\right)$. 0 intervalo $R R$ fetal, apresentou diferença significativa, quando comparado

Quadro 1. Índice APGAR para neonatos equinos segundo Smith (2006)

\begin{tabular}{|c|c|c|c|c|}
\hline & Parâmetros & 0 Ponto & 1 Ponto & 2 Pontos \\
\hline A & Atividade - tônus muscular & $\begin{array}{l}\text { Queda, decúbito } \\
\text { lateral }\end{array}$ & $\begin{array}{l}\text { Semi-esternal, alguma flexão } \\
\text { dos membros }\end{array}$ & Posição esternal \\
\hline $\mathrm{P}$ & Pulsação & Ausente & Abaixo de $60 \mathrm{bpm}$ & $60 \mathrm{bpm}$ ou mais \\
\hline $\mathrm{G}$ & $\begin{array}{l}\text { Expressão facial - estímulo nasal, } \\
\text { piparotes na orelha, estímulo } \\
\text { toracolombar }\end{array}$ & Sem resposta & $\begin{array}{l}\text { Expressão facial, movimento } \\
\text { da cabeça/pescoço }\end{array}$ & $\begin{array}{l}\text { Expressão facial forte, espirro, piparote na orelha, } \\
\text { sacudidas de cabeça, tentativa de ficar em estação } \\
\text { com movimentos de cabeça, pescoço e membros }\end{array}$ \\
\hline A & Aparência - cor das mucosas & Cinza/azuladas & Rósea clara & Rosada \\
\hline $\mathrm{R}$ & Respiração & Ausente & $<30$, irregular & $>30$, regular, relinchos \\
\hline
\end{tabular}

Pesq. Vet. Bras. 38(3):551-557, março 2018 


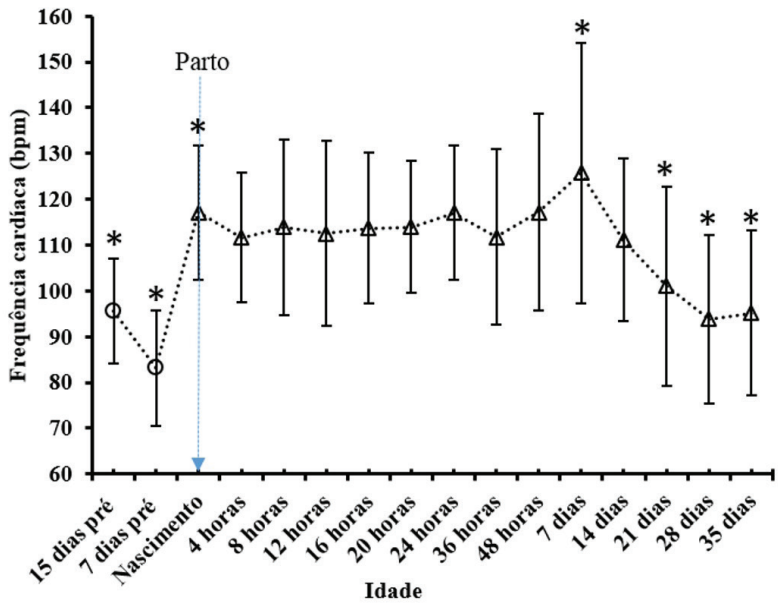

Fig.2. Frequência cardíaca fetal (o) 15 e sete dias pré-parto, e neonatal $(\Delta)$ com nascimento aos 35 dias, em equinos da raça Paint Horse.

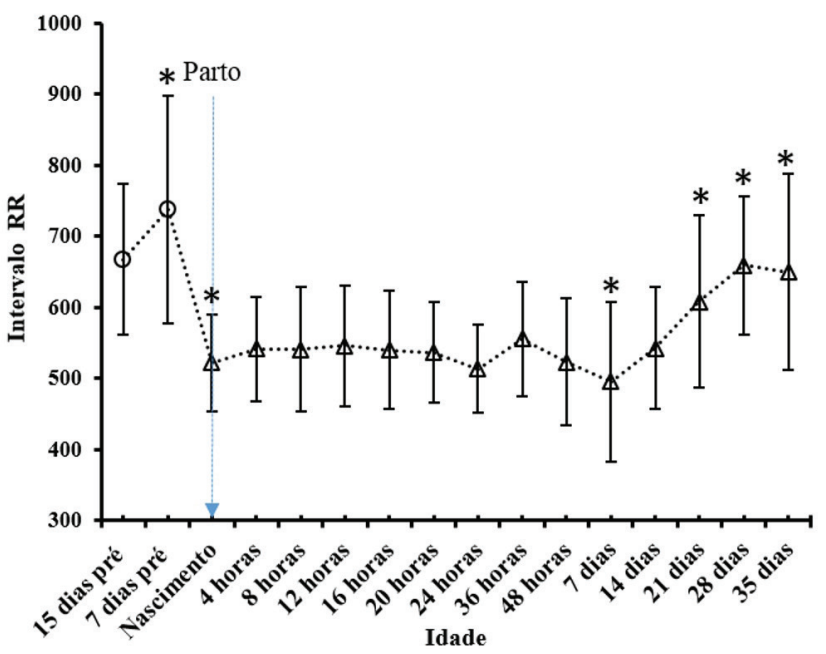

Fig.3. Intervalo RR fetal (O) 15 e sete dias pré-parto, e neonatal $(\Delta)$ com nascimento aos 35 dias, em equinos da raça Paint Horse.

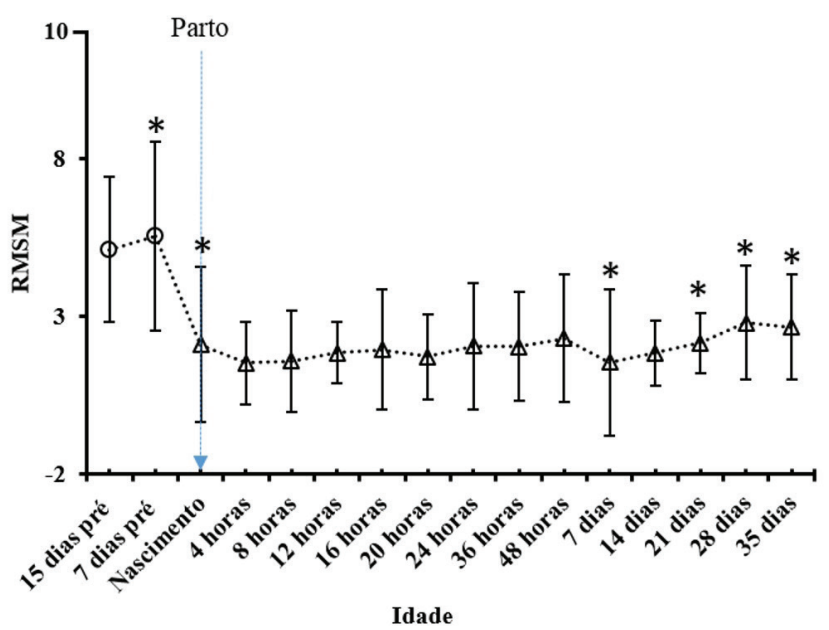

Fig.4. RMSM fetal (०) 15 e sete dias pré-parto, e neonatal $(\Delta)$ com nascimento aos 35 dias, em equinos da raça Paint Horse.

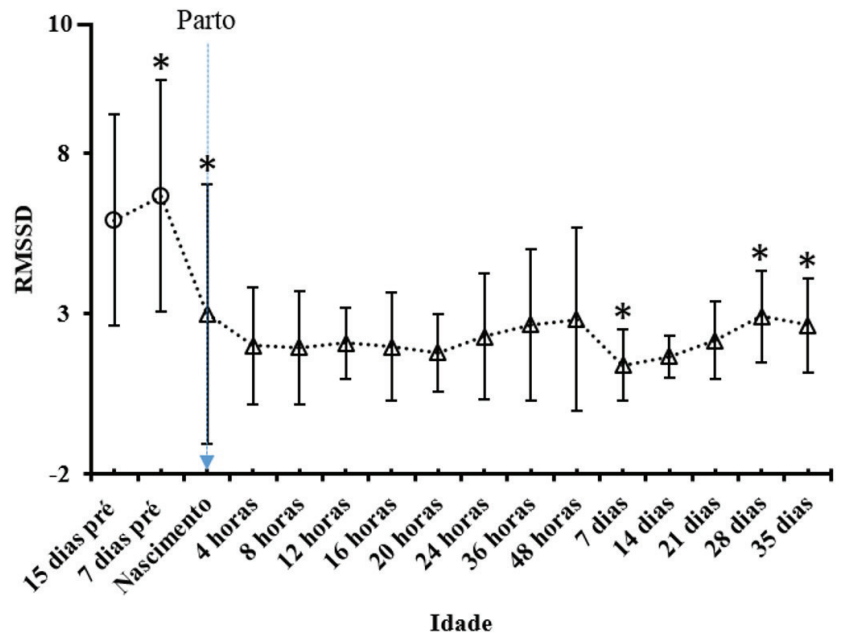

Fig.5. RMSSD fetal ( $(0) 15$ e sete dias pré-parto, e neonatal $(\Delta)$ com nascimento aos 35 dias, em equinos da raça Paint Horse.

aos neonatos até o $14^{\circ}$ dia; a partir do 21ํ dia foi semelhante, assim permanecendo até o 350 dia de idade.

Os resultados do escore de Apgar para os potros recém-nascidos deste estudo, expressos sob a forma de média e desvio padrão, demonstraram diferenças estatísticas entre os momentos analisados ( $\mathrm{p}<0,001)$, sendo $7,80 \pm 0,89$ a média ao nascimento e $8,35 \pm 0,99$ e aos 10 minutos.

Durante o período neonatal de potros da raça Paint Horse, a FC não diferiu significativamente entre o nascimento e os 14 dias de idade. A partir do nascimento, a FC manteve-se estável nas primeiras 48 horas. No sétimo dia, notou-se elevação deste parâmetro $(125,7 \pm 28,5 \mathrm{bpm})$, ocorrendo em seguida queda em seus valores. A FC ao nascimento $(117 \pm 14,6 \mathrm{bpm})$ diferiu significativamente, quando comparada ao $28^{\circ}(93,8 \pm 8,3 \mathrm{bpm})$ $(\mathrm{p}=0,0100)$ e $35^{\circ}$ dia de idade $(95,1 \pm 18,0 \mathrm{bpm})(\mathrm{p}=0,0008)$ (Fig.2).

O intervalo RR (Fig.3) permaneceu constante até 48 horas, sem diferença significativa entre os momentos. 0 menor valor encontrado foi aos sete dias de idade $(495,2 \pm 113,0 \mathrm{~ms})$, diferindo este momento do $21^{\circ}(p=0,0001), 28^{\circ}(p=0,0001)$ e $35^{\circ}$ dia $(p=0,0100)$, com estabilização a partir do $21^{\circ}$ dia.

O RMSM (Fig.4) apresentou comportamento constante durante todo o estudo; contudo para este índice, houve diferença significativa entre o sétimo, o $21^{\circ}(\mathrm{p}=0,0023)$, $28^{\circ}(p=0,0017)$ e $35^{\circ}$ dia $(p=0,0017)$. O RMSSD (Fig.5) apresentou diferença estatística aos sete dias, quando comparado ao $28^{\circ}(\mathrm{p}=0,0004)$ e $35^{\circ}$ dia $(\mathrm{p}=0,0006)$, sendo o maior valor ao nascimento $(2,98 \pm 4,03 \mathrm{~ms})$ e o menor aos sete dias $(1,38 \pm 1,10 \mathrm{~ms}) .0$ iTVV (Fig.6) sofreu um aumento gradual do nascimento $(3,96 \pm 12 \mathrm{~ms})$ até as 36 horas $(4,01 \pm 0,13 \mathrm{~ms})$, porém sem significância. Entre o sétimo $(3,9 \pm 0,19 \mathrm{~ms})$ e o $21^{\circ}(4,08 \pm 0,18)(\mathrm{p}<0,0001), 28^{\circ}(4,16 \pm 0,12 \mathrm{~ms})(\mathrm{p}<0,0001) \mathrm{e}$ 35 ㅇa $(4,14 \pm 0,17 \mathrm{~ms})(\mathrm{p}<0,0001)$ de idade, ocorreu elevação altamente significativa.

Os índices da VFC materna, fetal (15 e sete dias pré-parto) e neonatal (nas primeiras 48 horas pós-parto e semanalmente até os 35 dias de idade), em equinos da raça Paint Horse estão descritos no Quadro 2 sob a forma de média e desvio-padrão, nas Figuras 2, 3, 4, 5 e 6 e, o traçado eletrocardiográfico, na Figura 7. 
Quadro 2. Índices da variabilidade da frequência cardíaca materna, fetal (15 e sete dias pré-parto) e neonatal (nas primeiras 48 horas pós-parto e semanalmente até os 35 dias de idade), em equinos da raça Paint Horse

\begin{tabular}{|c|c|c|c|c|c|c|}
\hline & Horas/dias & $\mathrm{FC}(\mathrm{bpm})$ & Intervalo RR (ms) & RMSM (ms) & RMSSD (ms) & iTVV (ms) \\
\hline \multirow{2}{*}{$\begin{array}{l}\text { Dias pré-parto } \\
\text { (materno) }\end{array}$} & 15 dias & $58,9 \pm 10,9^{A}$ & $1048,3 \pm 167,8^{A}$ & $34,9 \pm 10,8^{\mathrm{A}}$ & $65,8 \pm 20,0^{A}$ & $4,6 \pm 0,2^{A}$ \\
\hline & 7 dias & $59,7 \pm 6,56^{A}$ & $1009,3 \pm 96,58^{A}$ & $32,8 \pm 11,9^{A}$ & $68,3 \pm 18,0^{A}$ & $4,5 \pm 0,08^{\mathrm{A}}$ \\
\hline \multirow{2}{*}{$\begin{array}{l}\text { Dias pré-parto } \\
\text { (fetal) }\end{array}$} & 15 dias & $95,6 \pm 11,4^{\mathrm{B}}$ & $667,0 \pm 106,1^{\text {в }}$ & $5,1 \pm 2,3^{\text {в }}$ & $5,9 \pm 3,3^{\mathrm{B}}$ & $4,2 \pm 0,1^{\text {в }}$ \\
\hline & 7 dias & $83,1 \pm 12,6^{\mathrm{C}}$ & $737,9 \pm 159,9^{\text {в }}$ & $5,5 \pm 3,0^{\text {в }}$ & $6,6 \pm 3,54^{\mathrm{B}}$ & $4,3 \pm 0,2^{\mathrm{B}}$ \\
\hline \multirow{9}{*}{$\begin{array}{l}\text { Horas pós-parto } \\
\text { (neonatal) }\end{array}$} & Nasc & $117,1 \pm 14,6^{\text {Dcd }}$ & $521,6 \pm 68,5^{\mathrm{Dab}}$ & $2,10 \pm 2,46^{\mathrm{Cab}}$ & $2,98 \pm 4,03^{\mathrm{Cab}}$ & $3,96 \pm 0,12^{\mathrm{Cab}}$ \\
\hline & 4 horas & $111,6 \pm 14,1^{\mathrm{Dbcd}}$ & $541,3 \pm 73,8^{\text {Dab }}$ & $1,51 \pm 1,31^{\mathrm{Cab}}$ & $1,99 \pm 1,83^{\mathrm{Cab}}$ & $3,99 \pm 0,12^{\mathrm{Cab}}$ \\
\hline & 8 horas & $113,9 \pm 19,1^{\text {Dcd }}$ & $540,9 \pm 87,5^{\text {Dab }}$ & $1,58 \pm 1,61^{\mathrm{Cab}}$ & $1,93 \pm 1,76^{\mathrm{Bab}}$ & $3,99 \pm 0,14^{\mathrm{Cab}}$ \\
\hline & 12 horas & $112,5 \pm 20,2^{\mathrm{Bbcd}}$ & $545,8 \pm 84,9^{\text {Dab }}$ & $1,84 \pm 0,97^{\mathrm{Cab}}$ & $2,08 \pm 1,11^{\text {Bab }}$ & $4,00 \pm 0,14^{\mathrm{Cab}}$ \\
\hline & 16 horas & $113,7 \pm 16,4^{\mathrm{Dcd}}$ & $539,8 \pm 82,7^{\text {Dab }}$ & $1,94 \pm 1,89^{\mathrm{Cab}}$ & $1,96 \pm 1,67^{\mathrm{Cab}}$ & $3,99 \pm 0,14^{\mathrm{Cab}}$ \\
\hline & 20 horas & $113,9 \pm 14,4^{\text {Dcd }}$ & $536,3 \pm 70,8^{\text {Dab }}$ & $1,72 \pm 1.35^{\mathrm{Cab}}$ & $1,77 \pm 1,21^{\mathrm{Cab}}$ & $3,98 \pm 0,11^{\mathrm{Cab}}$ \\
\hline & 24 horas & $117,0 \pm 14,7^{\text {Dcd }}$ & $513,4 \pm 62,3^{\mathrm{Da}}$ & $2,05 \pm 2,01^{\mathrm{Cab}}$ & $2,28 \pm 1,96^{\mathrm{Cab}}$ & $3,95 \pm 0,11^{\mathrm{Cab}}$ \\
\hline & 36 horas & $111,7 \pm 19,2^{\text {Dbcd }}$ & $555,7 \pm 80,2^{\text {Dab }}$ & $2,04 \pm 1,72^{\mathrm{Cab}}$ & $2,65 \pm 2,35^{\mathrm{Cab}}$ & $4,01 \pm 0,13^{\mathrm{Cab}}$ \\
\hline & 48 horas & $117,1 \pm 21,5^{\text {Dcd }}$ & $523,0 \pm 89,5^{\text {Dab }}$ & $2,29 \pm 2,02^{\mathrm{Cab}}$ & $2,80 \pm 2,85^{\mathrm{Cab}}$ & $3,96 \pm 0,15^{\mathrm{Ca}}$ \\
\hline \multirow{5}{*}{$\begin{array}{l}\text { Dias pós-parto } \\
\text { (neonatal) }\end{array}$} & 7 dias & $125,7 \pm 28,5^{\text {Dd }}$ & $495,2 \pm 113,0^{\mathrm{Da}}$ & $1,54 \pm 2,32^{\mathrm{Ca}}$ & $1,38 \pm 1,10^{\mathrm{Ca}}$ & $3,90 \pm 0,19^{\mathrm{Ca}}$ \\
\hline & 14 dias & $111,1 \pm 17,7^{\text {Dbcd }}$ & $542,6 \pm 86,2^{\text {Dab }}$ & $1,83 \pm 1,04^{\mathrm{Cab}}$ & $1,65 \pm 0,64^{\mathrm{Cab}}$ & $3,99 \pm 0,14^{\mathrm{Cab}}$ \\
\hline & 21 dias & $101,0 \pm 21,7^{\text {Dabc }}$ & $607,6 \pm 121,3^{\mathrm{Bbc}}$ & $2,15 \pm 0,96^{\mathrm{Cb}}$ & $2,15 \pm 1,21^{\mathrm{Cab}}$ & $4,08 \pm 0,18^{\mathrm{Cbc}}$ \\
\hline & 28 dias & $93,8 \pm 18,3^{\text {Ва }}$ & $659,0 \pm 97,2^{\mathrm{Bc}}$ & $2,78 \pm 1,80^{\mathrm{cb}}$ & $2,90 \pm 1,43^{\mathrm{Cb}}$ & $4,16 \pm 0,12^{\mathrm{Bc}}$ \\
\hline & 35 dias & $95,1 \pm 18,0^{\text {Bab }}$ & $649,1 \pm 137,9^{\mathrm{Bc}}$ & $2,65 \pm 1,67^{\mathrm{Cb}}$ & $2,62 \pm 1,45^{\mathrm{Cb}}$ & $4,14 \pm 0,17^{\mathrm{Bc}}$ \\
\hline
\end{tabular}

Letras minúsculas diferentes $(a, b)$ presentes na mesma coluna, indicam diferença significativa entre os momentos $(a \neq b$ : $p<0,05)$. Letras maiúsculas $(A, B)$ indicam diferença significativa entre as diferentes faixas etárias (materna, fetal e neonatal) $(A \neq B: p<0,05)$. $F C=$ frequência cardíaca, bpm $=$ batimentos por minutos, $\mathrm{ms}=$ milissegundos, $\mathrm{mV}=$ milivolts.

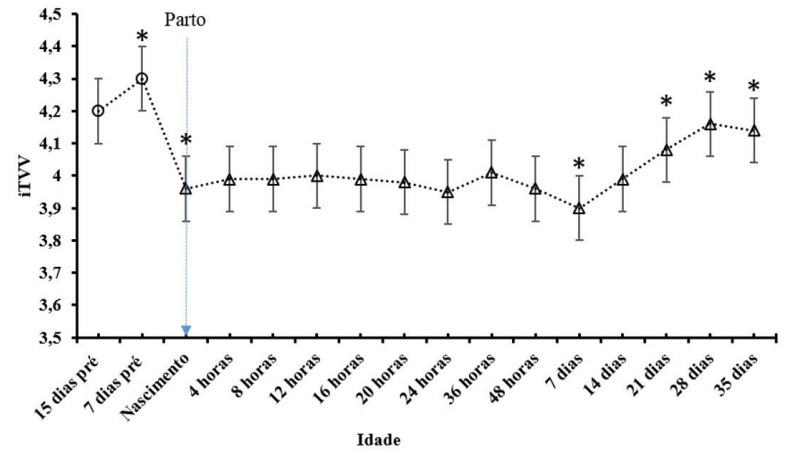

Fig.6. iTVV fetal (०) 15 e sete dias pré-parto, e neonatal $(\Delta)$ com nascimento aos 35 dias, em equinos da raça Paint Horse.

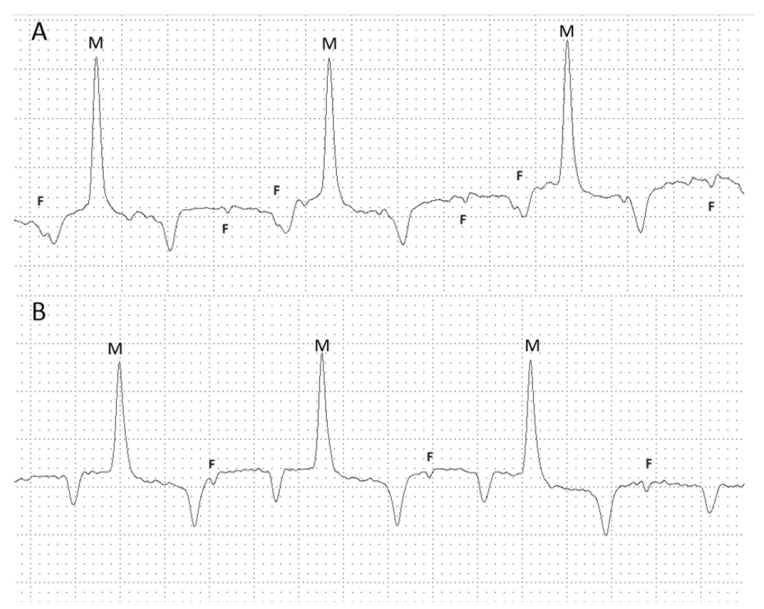

Fig.7. Exemplo de traçado eletrocardiográfico materno e fetal de um equino aos sete (A) e 15 dias (B) pré-parto.

\section{DISCUSSÃO}

A avaliação da VFC é uma técnica não invasiva que pode ser utilizada para investigar o funcionamento do SNA, especialmente o balanço entre a atividade vagal e simpática, bem como estresse e bem-estar animal (Von Borell et al. 2007).

A FC e VFC materna se mantiveram estáveis durante os 15 e sete dias pré-parto, não sendo adequados como preditores da proximidade do mesmo, bem como não foi detectado nenhum distúrbio, fator estressante ou injúria na égua prenhe que promovesse alteração dos índices, durante este período de avaliação. Diminuições na VFC em equinos adultos estão associadas ao estresse (Schmidt et al. 2010). Os resultados obtidos neste estudo foram semelhantes aos descritos por outros autores (Nagel et al. 2011, 2012), que também concluíram que nos últimos 10 dias previamente ao parto, a FC e a VFC não se alteram na égua.

A média da FCF diminuiu significativamente entre os $15 \mathrm{e}$ os sete dias pré-parto, estando os valores dentro dos obtidos para fetos saudáveis por Nagel et al. (2010), entretanto os índices de VFC não se alteraram nos fetos desta raça, sugerindo bem-estar e viabilidade fetal. Van Leeuwen et al. (2007) descrevem que a VFC é influenciada pela FC, e seus valores elevados geralmente são associados a uma menor VFC fetal, entretanto, essa queda na VFC não foi observada no presente estudo.

Pode-se observar durante o período fetal, um predomínio parassimpático, demonstrado tanto pela frequência cardíaca inferior à neonatal, como também pelos índices da VFC (intervalo RR, RMSM, RDSSD e iTVV) significativamente superiores aos neonatais. Contudo este comportamento pode revelar não apenas predomínio parassimpático (Van Leeuwen et al. 1999, Schneider et al. 2008), como também 
imaturidade do sistema simpático previamente ao parto em fetos equinos da raça Paint Horse.

Ao nascimento, o escore modificado de Apgar foi inferior ao realizado aos 10 minutos após. A média encontrada manteve-se entre sete e oito nos dois momentos estudados, sendo considerada adequada, indicando boa viabilidade neonatal (Lu et al. 2006, Paradis 2006). Entretanto, para alguns autores (Vaala et al. 2006), escores entre seis e oito indicam a presença de asfixia moderada com sugestão da adoção de medidas de reanimação neonatal. Os valores desse escore devem ser propostos segundo a raça e tempo de realização, visto que os animais deste estudo não apresentaram alterações clínicas condizentes com qualquer grau de asfixia.

Durante as primeiras 48 horas de vida, observou-se diferença significativa entre fetos e neonatos, demonstrando inversão do predomínio autonômico parassimpático (fetal) para o simpático, na fase neonatal. 0 neonato apresenta valores baixos de pressão arterial, volume sanguíneo e resistência vascular periférica, de forma que, para manter uma perfusão adequada, mantém maior frequência e debito cardíacos, bem como pressão venosa central, em comparação aos adultos (Magrini 1978, Adelman \& Wright 1985). A maturação pós-natal do controle da circulação central, do débito cardíaco e do sistema vascular periférico, assim como mudanças de desenvolvimento no nodo sinusal (McDonald 1980, Gootman et al. 1981, Blanco et al. 1988) alteram a frequência cardíaca e VFC. Com isto, sugere-se que neste período os valores reduzidos nos índices de VFC são justificados não por inviabilidade do potro recém-nascido da raça Paint Horse, mas sim devido a predominância do ramo simpático, necessária frente às demandas do sistema biológico e manutenção da homeostase.

De acordo com os resultados deste estudo, houve diferença significativa dos parâmetros maternos quando comparados aos fetais e neonatais, sendo os primeiros, superiores em relação aos índices da VFC, em todos os momentos da análise, indicando que o potro neonato desta raça, até seus 35 dias de vida ainda não possui maturidade autonômica, quando comparado ao adulto. Entretanto nas últimas semanas neonatais, os valores da FC (aos 28 e 35 dias), do intervalo RR (aos 21, 28 e 35 dias) e do iTVV (aos 28, 35 dias), aproximam-se aos encontrados no período fetal, indicando um início do equilíbrio entre os ramos do sistema nervoso autônomo a partir da terceira e/ou quarta semanas de vida, demonstrando adaptação ou amadurecimento frente as demandas do sistema biológico e regulação da homeostase.

De acordo com Von Borell et al. (2007), o RMSSD é a medida primária no domínio do tempo utilizada para representar a atividade regulatória vagal. Dessa forma é provável que a partir do $28^{\circ}$ dia, período no qual esse índice tende a aumentar significativamente, seja início do equilíbrio deste sistema no neonato equino da raça Paint Horse.

\section{CONCLUSÕES}

A frequência cardíaca (FC) e a variabilidade da frequência cardíaca (VFC) fetal e materna não foram preditores fidedignos da proximidade do parto em equinos da raça Paint Horse, entretanto, o eletrocardiograma materno-fetal mostrou-se como uma técnica confiável para detectar sinais cardíacos em fetos equinos no terço final da prenhez.

Os resultados obtidos da VFC fetal e neonatal do presente estudo, quando comparados aos de um equino adulto, indicam o predomínio parassimpático durante a fase fetal e simpático durante a neonatal, até a terceira e/ou quarta semanas de idade, momento no qual se inicia a modulação entre os dois sistemas.

\section{REFERÊNCIAS}

Adams-Brendemuehl C. \& Pipers F.S. 1987. Antepartum evaluations of the equine fetus. J. Reprod. Fertil. Suppl. 35:565-573. PMid:3316646.

Adelman R.D. \& Wright J. 1985. Systolic blood pressure and heart rate in the growing beagle puppy. Dev. Pharmacol. Ther. 8(6):396-401. http://dx.doi. org/10.1159/000457064. PMid:4075938.

Aubert A.E., Seps B. \& Beckers F. 2003. Heart rate variability in athletes. Sports Med. 33(12):889-919. http://dx.doi.org/10.2165/00007256200333120-00003. PMid:12974657.

Baska-Vincze B., Baska F. \& Szenci 0. 2015. Fetal heart rate and fetal heart rate variability in Lipizzaner broodmares. Acta Vet. Hung. 63(1):89-99. http://dx.doi.org/10.1556/AVet.2015.007. PMid:25655416.

Blanco C.E., Dawes G.S., Hanson M.A. \& Mccooke H.B. 1988. Carotid baroreceptors in fetal and newborn sheep. Pediatr. Res. 24(3):342-346. http://dx.doi. org/10.1203/00006450-198809000-00014. PMid:3211620.

Bocking A.D. 2003. Assessment of fetal heart rate and fetal movements in detecting oxygen deprivation in-utero. Eur. J. Obstet. Gynecol. Reprod. Biol. 110(Suppl.1):S108-S112. http://dx.doi.org/10.1016/S0301-2115(03)001805. PMid:12965098.

Brito F.S. 2009. Eletrocardiografia ambulatorial: sistema Holter. Arq. Bras. Cardiol. 93(6, Supl.2):e179-e264. PMid:20676461.

Carareto R., Sousa M.G., Zacheu J.C., Aguiar A.J.A. \& Camacho A.A. 2007. Variabilidade da frequência cardíaca em cães anestesiados com infusão contínua de propofol e sufentanil. Arq. Bras. Med. Vet. Zootec. 59(2):329332. http://dx.doi.org/10.1590/S0102-09352007000200009.

Dawes G.S., Moulden M. \& Redman C.W. 1992. Short-term fetal heart rate variation, decelerations, and umbilical flow velocity waveforms before labor. Obstet. Gynecol. 80(4):673-678. PMid:1407893.

Dennis A., Arhanghelschi I., Simmons S. \& Royse C. 2010. Prospective observational study of serial cardiac output by transthoracic echocardiography in healthy pregnant women undergoing elective caesarean delivery. Int. J. Obstet. Anesth. 19(2):142-148. http://dx.doi.org/10.1016/j.ijoa.2009.06.007. PMid:20202818.

Gootman P.M., Gootmant N., Turlapaty P.D.M.V., Yao A.C., Buckleyt B.J. \& Altura B.M. 1981. Autonomic regulation of cardiovascular function in neonates, p.70-93. In: Ibid. (Eds), Development of the Autonomic Nervous System. Pitman Medical, London. http://dx.doi.org/10.1002/9780470720653.ch4.

Häggström J., Hamlin R.L., Hansson K. \& Kvart C. 1996. Heart rate variability in relation to severity of mitral regurgitation in Cavalier King Charles spaniels. J. Small Anim. Pract. 37(2):69-75. http://dx.doi.org/10.1111/j.1748-5827.1996. tb01941.x. PMid:8656596.

Lu K.G., Barr B.S., Embertson R. \& Schaer B.D. 2006. Dystocia: a true equine emergency. Clin. Tech. Equine Pract. 5(2):145-153. http://dx.doi. org/10.1053/j.ctep.2006.03.008.

Magrini F. 1978. Haemodynamic determinants of the arterial blood pressure rise during growth in conscious puppies. Cardiovasc. Res. 12(7):422-428. http://dx.doi.org/10.1093/cvr/12.7.422. PMid:719655.

Manning F.A. 2002. Fetal biophysical profile: a critical appraisal. Clin. Obstet. Gynecol. 45(4):975-985. http://dx.doi.org/10.1097/00003081-20021200000004. PMid:12438875.

McDonald A.H. 1980. Mechanisms affecting heart-rate, p.3-12. In: Kitney R.I. \& Rompelman O. (Eds), The Study of Heart-rate Variability. Clarendon Press, Oxford. 
Nagel C., Aurich J. \& Aurich C. 2010. Determination of heart rate and heart rate variability in the equine fetus by fetomaternal electrocardiography. Theriogenology 73(7):973-983. http://dx.doi.org/10.1016/j. theriogenology.2009.11.026. PMid:20106514.

Nagel C., Aurich J. \& Aurich C. 2011. Heart rate and heart rate variability in the pregnant mare and its foetus. Reprod. Domest. Anim. 46(6):990-993. http://dx.doi.org/10.1111/j.1439-0531.2011.01772.x. PMid:21382105.

Nagel C., Erber R., Bergmaier C., Wulf M., Aurich J., Möstl E. \& Aurich C. 2012. Cortisol and progestin release, heart rate and heart rate variability in the pregnant and postpartum mare, fetus and newborn foal. Theriogenology 78(4):759-767. http://dx.doi.org/10.1016/j.theriogenology.2012.03.023. PMid:22626780.

Nagel C., Erber R., Ille N., Von Lewinski M., Aurich J., Möstl E. \& Aurich C. 2014. Parturition in horses is dominated by parasympathetic activity of the autonomous nervous system. Theriogenology 82(1):160-168. http:// dx.doi.org/10.1016/j.theriogenology.2014.03.015. PMid:24767599.

Nogueira C.E.W. \& Lins L.A. 2009. Neonatologia e pediatria equina. Vol I. Editora UFPel, Pelotas. 168p.

Paradis M.R. 2006. Equine neonatal medicine: a case based approach. Elsevier, Philadelphia. 286p.

Pereira E.Z. 2011. Contribuição das eletrocardiografias convencional, Holter e de alta resolução no diagnóstico da cardiomiopatia arritmogênica do ventrículo direito dos cães da raça Boxer. Dissertação de Mestrado em Clinica Médica Veterinária, Faculdade de Ciências Agrárias e Veterinárias, Unesp, Jaboticabal. 37p.

Pumprla J., Howorka K., Groves D., Chester M. \& Nolan J. 2002. Functional assessment of heart rate variability: physiological basis and practical applications. Int. J. Cardiol. 84(1):1-14. http://dx.doi.org/10.1016/S01675273(02)00057-8. PMid:12104056.

Schmidt A., Möstl E., Wehnert C., Aurich J., Müller J. \& Aurich C. 2010. Cortisol release and heart rate variability in horses during road transport. Horm. Behav. 57(2):209-215. http://dx.doi.org/10.1016/j.yhbeh.2009.11.003. PMid:19944105.

Schneider U., Frank B., Fiedler A., Kaehler C., Hoyer D., Liehr M., Haueisen J. \& Schleussner E. 2008. Human fetal heart rate variability: characteristics of autonomic regulation in the third trimester of gestation. J. Perinat. Med. 36(5):433-441. http://dx.doi.org/10.1515/JPM.2008.059. PMid:18605969.

Siimes A.S.I., Välimäki I.A.T., Antila K.J., Julkunen M.K.A., Metsala T.H., Halkola L.T. \& Samuli Sarajas H.S. 1990. Regulation of heart rate variation by the autonomic nervous system in neonatal lambs. Pediatr. Res. 27(4):383-391. http://dx.doi.org/10.1203/00006450-199004000-00012. PMid:2160636.
Smith B.P. 2006. Medicina interna de grandes animais. Manole, Davis. 1728p.

Tárraga K.M., Spinosa H.S. \& Camacho A.A. 2000. Electrocardiographic evaluation of two anesthetic combinations in dogs. Arq. Bras. Med. Vet. Zootec. 52(2):138-143. http://dx.doi.org/10.1590/S0102-09352000000200009.

Task Force. 1996. Heart Rate Variability: standards of measurement, physiological interpretation and clinical use. Circulation 93(5):1043-1065. http://dx.doi.org/10.1161/01.CIR.93.5.1043. PMid:8598068.

Trenk L., Kuhl J., Aurich J., Aurich C. \& Nagel C. 2015. Heart rate and heart rate variability in pregnant dairy cows and their fetuses determined by fetomaternal electrocardiography. Theriogenology 84(8):1405-1410. http://dx.doi.org/10.1016/j.theriogenology.2015.07.027. PMid:26279313.

Vaala W.E., House J.K. \& Madigan J.E. 2006. Conduta inicial e exame físico do neonato, p.277-293. In: Smith B.P. (Ed), Medicina interna de grandes animais. $3^{\mathfrak{a}}$ ed. Manole, São Paulo.

Van Leeuwen P., Lange S., Bettermann H., Grönemeyer D. \& Hatzmann W. 1999. Fetal heart rate variability and complexity in the course of pregnancy. Early Hum. Dev. 54(3):259-269. http://dx.doi.org/10.1016/ S0378-3782(98)00102-9. PMid:10321792.

Van Leeuwen P., Lange S., Geue D. \& Grönemeyer D. 2007. Heart rate variability in the fetus: a comparison of measures. Biomed. Tech. 52(1):61-65. PMid:17313336.

Vintzileos A.M., Fleming A.D., Scorza W.E., Wolf E.J., Balducci J., Campbell W.A. \& Rodis J.F. 1991. Relationship between fetal biophysical activities and umbilical cord blood gas values. Am. J. Obstet. Gynecol. 165(3):707713. http://dx.doi.org/10.1016/0002-9378(91)90314-H. PMid:1822963.

Von Borell E., Langbein J., Després G., Hansen S., Leterrier C., Marchant-Forde J., Marchant-Forde R., Minero M., Mohr E., Prunier A., Valance D. \& Veissier I. 2007. Heart rate variability as a measure of autonomic regulation of cardiac activity for assessing stress and welfare in farm animals, a review. Physiol. Behav. 92(3):293-316. http://dx.doi.org/10.1016/j.physbeh.2007.01.007. PMid:17320122.

Wheeler T., Cooke E. \& Murrills A. 1979. Computer analysis of fetal heart rate variation during normal pregnancy. Brit. J. Obstet. Gynaecol. 86(3):186-197. http://dx.doi.org/10.1111/j.1471-0528.1979.tb10592.x. PMid:427060.

Woods Junior J.R., Dandavino A., Murayama K., Brinkman 3rd C.R. \& Assali N.S. 1977. Autonomic control of cardiovascular functions during neonatal development and in adult sheep. Circ. Res. 40(4):401-407. http://dx.doi. org/10.1161/01.RES.40.4.401. PMid:14791. 